Please quote as: Leimeister, J. M. (2009): IT-basierte, gemeinschaftsgestützte Innovationsentwicklung für Softwareunternehmen. Hrsg./Editors: Spath, D. Verlag/Publisher: Fraunhofer-Verlag, Stuttgart, Germany. Erscheinungsjahr/Year: 2009. 


\title{
IT-basierte, gemeinschaftsgestützte Innovationsentwicklung für Softwareunternehmen
}

\author{
Jan Marco Leimeister \\ Universität Kassel, Lehrstuhl für Wirtschaftsinformatik
}

\begin{abstract}
Im internationalen Vergleich liegt die Innovationstätigkeit von deutschen Softwareunternehmen nicht auf Spitzenniveau. Arbeits- und Dienstleistungsforschung kann hierbei als wichtiger Innovationstreiber fungieren. Im folgenden Beitrag wird als Beispiel hierfür das BMBF-geförderte Forschungsprojekt „Gemeinschaftsgetützte Innovationsentwicklung für Softwareunternehmen" (GENIE) ${ }^{1}$ vorgestellt, das durch intelligenten IT-Einsatz Open Innovation-Konzepte in die Softwareindustrie trägt. Ziel dieses Projektes ist es, die Innovationsentwicklung von Softwareunternehmen durch Innovationscommunities für Kunden und andere Wertschöpfungspartner nachhaltig zu stärken. Auf der Basis empirisch und konzeptionell gewonnener Erkenntnisse wird das Konzept dieser GENIE-Communities veranschaulicht sowie weiterer Forschungsbedarf für das Themenfeld Innovationsentwicklung \& IT aufgezeigt.
\end{abstract}

\section{Treiber und Quellen von Innovationen - Open Innovation und Wisdom of Crowds}

"Large groups of people are smarter than an elite few, no matter how brilliant - better at solving problems, fostering innovation, coming to wise decisions, even predicting the future."

(Surowiecki 2005)

Die Innovationsfähigkeit eines Unternehmens hängt heute maßgeblich davon ab, Kunden und andere Wertschöpfungspartner in das Innovationsmanagement zu integrieren, denn von diesen Anspruchsgruppen geht auf Grund ihrer Produkterfahrung und ihres Produktverständnisses ein großes, oft noch nicht gehobenes Innovationspotenzial aus. Kunden sind heute nicht mehr nur noch passive Empfänger und Konsumenten in einer von Herstellern dominierten Wertschöpfung. Sie gestalten vielmehr Produkte und Dienstleistungen aktiv mit und übernehmen dabei sogar teilweise

\footnotetext{
${ }^{1}$ Das Verbundvorhaben GENIE - Gemeinschaftsgestützte Innovationsentwicklung für Softwareunternehmen wird vom BMBF unter Förderkennzeichen: 01FM07027 gefördert und vom Projektträger DLR - Arbeitsgestaltung und Dienstleistung fachlich betreut. Für weitergehende Informationen siehe http://projekt-genie.de/.
} 
Entwicklung und Herstellung der nachgefragten Produkte (Reichwald, Piller 2006; von Hippel 2005).

Diese interaktive Wertschöpfung ist eine Abkehr vom klassischen Innovationsprozess, der sich weitgehend innerhalb der eignen Unternehmensgrenzen abspielt und in dem ausschließlich Ideen aus unternehmensinternen Bereichen, insbesondere der Forschung und Entwicklung, kommerzialisiert werden (Reichwald, Piller 2006). In offenen Innovationsprozessen bringen Unternehmen jedoch sowohl eigene als auch unternehmensfremde Ideen zur Marktreife bzw. entwickeln Wege Innovationen auch außerhalb der Unternehmensgrenzen, bspw. in strategischen Allianzen, zu einem Erfolg zu führen (Chesbrough 2003). Dieser vielschichtige und offene Such- und Lösungsprozess wird in Abbildung 1 dargestellt.

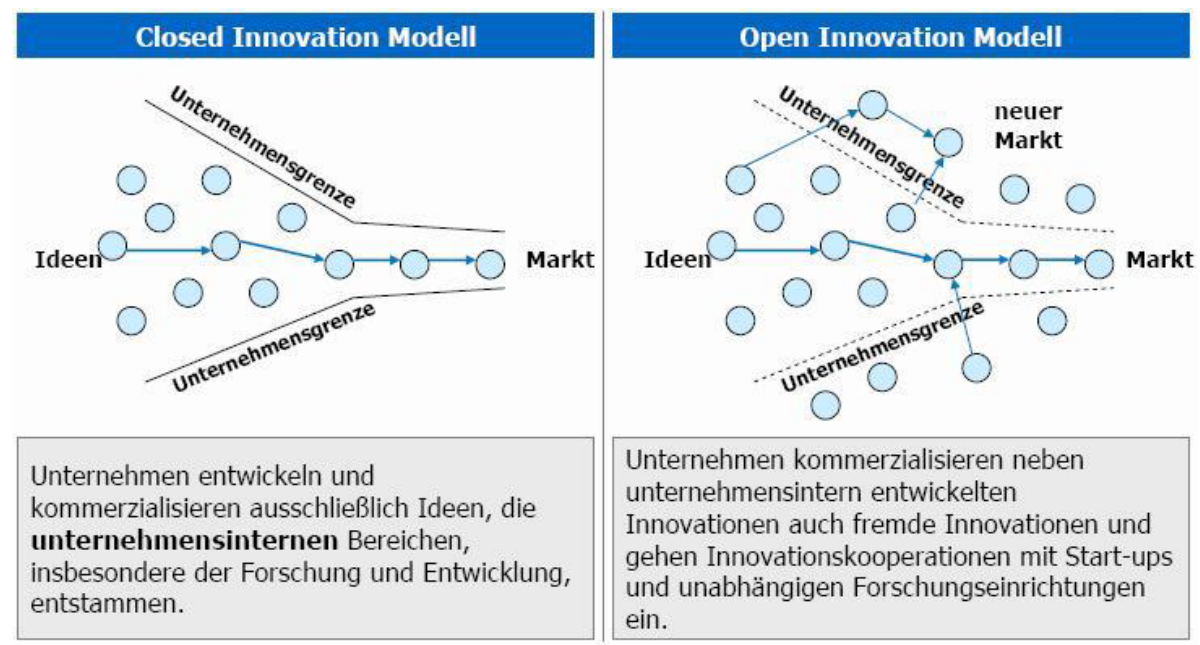

Abbildung 1: $\quad$ Gegenüberstellung von Open und Closed Innovation (Quelle: Reichwald, Piller 2006 angelehnt an Chesbrough 2003)

Eines der dem Open Innovation Ansatz zugrundeliegenden Prinzipien ist das Nutzbarmachen der sog. „Weisheit der Vielen“. Dieses Prinzip der kollektiven Intelligenz besagt, dass innerhalb einer sozialen Gemeinschaft durch den Beitrag jedes Einzelnen bessere Entscheidungen erzeugt werden können als durch einzelne Experten oder Individuen (Lévy 1997). Open Innovation knüpft genau an diesem Punkt an. Durch die Öffnung des Innovationsprozesses für externe Einflüsse und die Auslagerung von Aufgaben an die Akteure, die besondere Kompetenzen oder Wissen zu ihrer Lösung besitzen, können Unternehmen zahlreiche Vorteile erschließen: kürzere Innovationszyklen, Kostenreduktion im Entwicklungsprozess, Erhöhung der eigenen Innovationskraft, Erschließung neuer Märkte und Umsatzpotentiale sowie die Reduktion von marktlicher und technologischer Unsicherheit im Innovationsprozess (Chesbrough 2007; Chesbrough, Schwartz 2007; Gassmann, Enkel 2004; Reichwald, Piller 2006).

\section{Innovationstätigkeit der deutschen Softwareindustrie}

Die Innovationsstärke in Deutschland nimmt für die Bereiche Maschinen und Industriegüter im Weltmarktvergleich eine sehr gute Stellung ein. Als herausragendes Beispiel kann hier die deutsche Automobilindustrie genannt werden, der es durch ein 
ausgeprägtes Innovationsverhalten gelingt, ihre Position im Weltmarkt zu behaupten (Holl et al. 2006).

Dies gilt jedoch nicht für die deutschen Softwareunternehmen. Im Bereich der Entwicklung von Softwareprodukten nehmen deutsche Firmen im Weltmarktvergleich nur mittlere Positionen ein (Wohlrab 2003; BCG 2006).

Deutschen Softwareunternehmen fehlt es oftmals an einer entsprechenden Innovationskultur und einem systematischen Innovationsmanagement. Die Ideenfindung erfolgt meist informell, ohne Nachhaltigkeit und ist quasi dem Zufall überlassen (Holl et al. 2006). Als Hauptlieferant für die Ideenproduktion gelten vor allem die eigenen Mitarbeiter (Koch, Möslein 2007; Holl et al. 2006), wobei hilfreiche Werkzeuge wie z.B. ein betriebliches Vorschlagswesen nur sehr gering verbreitet sind. Als zweite Quelle von Innovationsideen ist weiterhin die Erfassung von Kundenwünschen üblich, die in der Regel über Kundenbetreuer, den Support, Gespräche mit Fachhändlern oder Marktbeobachtungen unsystematisch erfasst und anschließend intern zu möglichen Anforderungswünschen ausformuliert werden (Holl et al. 2006).

Diese unsystematische Erhebung von Bedürfnisinformationen birgt die Gefahr, dass Softwareprodukte an den Bedürfnissen des Kunden „Vorbei innoviert“ werden. Immer wieder werden technisch ausgefeilte und aus unternehmensinterner Sicht hoch attraktive Produkte auf den Markt gebracht, die aber die Erwartungen der Kunden nicht erfüllen. Die Kunden werden in diesem Umfeld eher als Wertempfänger der veräußerten Produkte anstatt als Innovationsquelle begriffen. Aber auch Partnerschaften mit dem Ziel einer gemeinschaftlichen Produktentwicklung sind in der Softwarebranche eher unüblich (Holl et al. 2006) wenngleich in anderen Branchen die Kooperation in virtuellen Verbünden weiter fortgeschritten sind. Im Allgemeinen sind die wenig systematischen Innovationstätigkeiten der deutschen Softwareindustrie sehr stark nach innen gerichtet. Obwohl erfolgreiche Ansätze aus dem „Open Source-Bereich“ das große Potential von offenen Innovationsprozessen für die Softwareentwicklung aufzeigen (Lakhani, von Hippel 2003; Piller 2003; von Krogh et al. 2003), kommen diese in der deutschen Softwarebranche nur sporadisch zum Einsatz.

In der Folge besitzen deutsche Softwareunternehmen oftmals nur eine geringe Innovationskraft, so dass häufig nur inkrementelle Innovationen hervorgebracht werden können (Koch, Möslein 2007). Bestehende Softwareprodukte werden nur einer Weiterentwickelung unterzogen, große Innovationssprünge sind dagegen selten zu beobachten. Viele Softwarefirmen konzentrieren sich auf die Weiterentwicklung ihrer im Laufe der Zeit veralternden Software anstatt sich auf die Entwicklung neuer Produkte zu konzentrieren. 


\section{IT-basierte, gemeinschaftsgestützte Innovations- entwicklung als Lösungsansatz - Das Forschungs- projekt GENIE}

\subsection{Ziele von GENIE}

Grundgedanke des Projektes "Gemeinschaftsgetützte Innovationsentwicklung für Softwareunternehmen" (GENIE) ist die Schaffung von Innovationscommunities, deren Mitglieder sich aus Wertschöpfungspartnern und anderen Anspruchsgruppen (insbesondere Kunden, Forschungs- und Projektpartner, etc.) eines Softwareunternehmens zusammensetzen. Gemeinsames Ziel einer solchen Community soll es sein, gemeinschaftlich sowohl auf einer zentralen Internetplattform als auch in realweltlichen Settings Innovationsideen zu generieren sowie diese in Zusammenarbeit zu konkreten Innovationsprojekten zu verdichten, die bei ausreichendem Marktpotential in Prototypen oder Business-Plänen münden können. Auf dieser Basis entstehen innovative Produkte, die dem initiierenden Unternehmen neue Möglichkeiten in neuen oder bereits bestehenden Märkten eröffnen. Das Innovationsmanagement von Softwareunternehmen wird systematisiert, die Innovationsaktivitäten nach außen geöffnet sowie externe Innovationspotenziale im gesamten Innovationsprozess nutzbar gemacht (Bretschneider et al. 2008). Eine solche GENIE-Community ist in Abbildung 2 dargestellt.

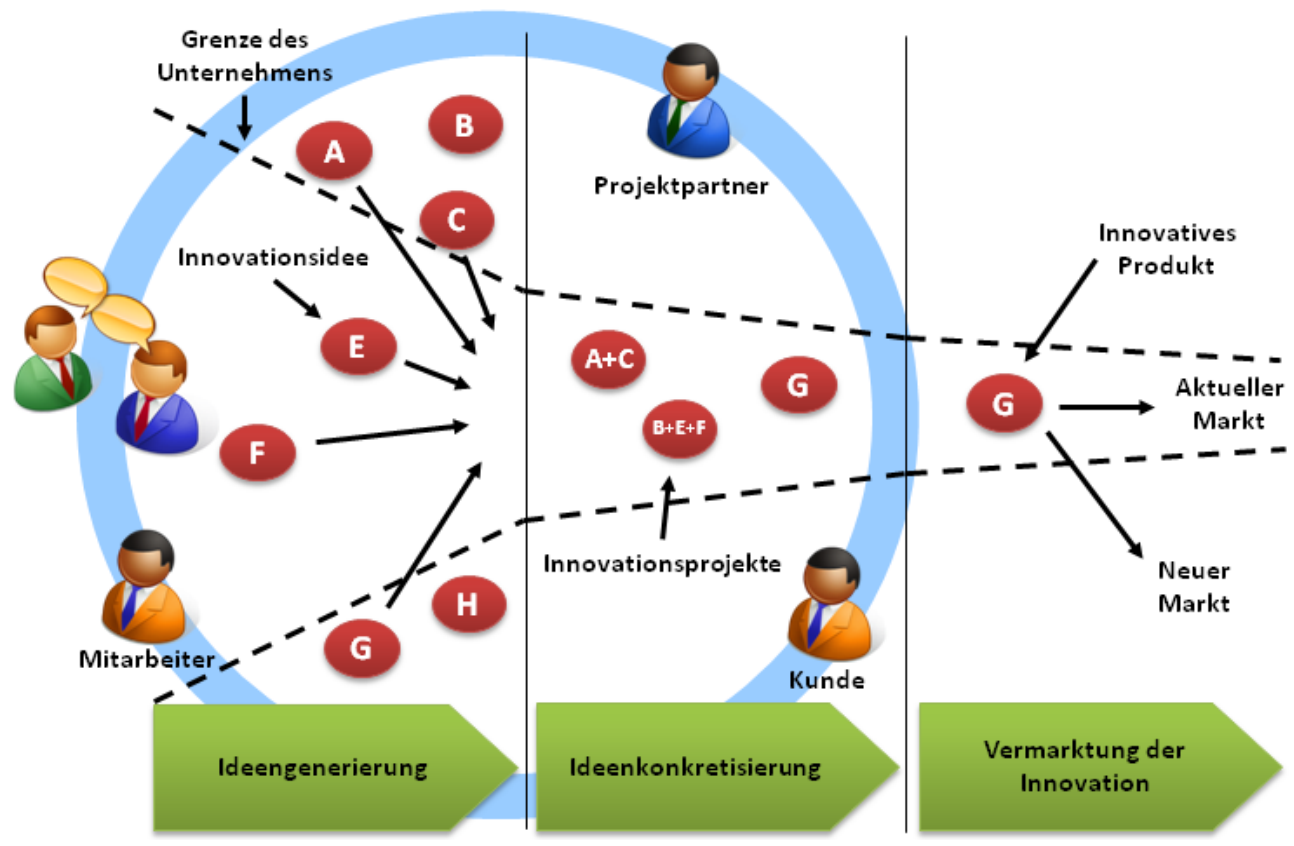

Abbildung 2: $\quad$ Das Konzept der GENIE-Community (Quelle: Eigene Darstellung)

Dem GENIE-Konzept liegt ein Ideenevolutionsprozess zu Grunde, der den Reifeprozess einer Idee entlang des Innovationsprozesses beschreibt (vgl. Abbildung 3). In der ersten Teilphase der „Ideengenerierung“, also der frühen Phase des Innovationsprozesses, werden von den Community-Mitgliedern Ideen vorgeschlagen. Diese werden dann in der Community durch Kommentierung und Kombination mit den Ideen anderer Community-Mitglieder schrittweise verfeinert. Auf diese Weise entste- 
hen durch die Vernetzungs- und Kollaborationsaktivitäten der Community-Mitglieder aus den Ursprungsideen sukzessiv konkretere und aussagekräftigere Innovationsideen.

In der zweiten Phase der „Ideenimplementierung“ werden die Ideen entweder durch die Community selbst oder das initiierende Unternehmen bewertet und im Rahmen einer weiteren kollaborativen Bearbeitung sukzessive mit Lösungsinformationen angereichert, d.h. die Ideen gewinnen in immer stärkerem Ausmaß an Ausarbeitungsgrad sowie an Informationen darüber, wie sie unter Anwendung von Wissen, Technologien und Fertigungstechniken umgesetzt werden können (von Hippel 1978, 1994). Durch diese kollaborative Elaboration zwischen implementierendem Unternehmen und der Community werden die Innovationsideen zu konkreten Innovationsprojekten getragen.
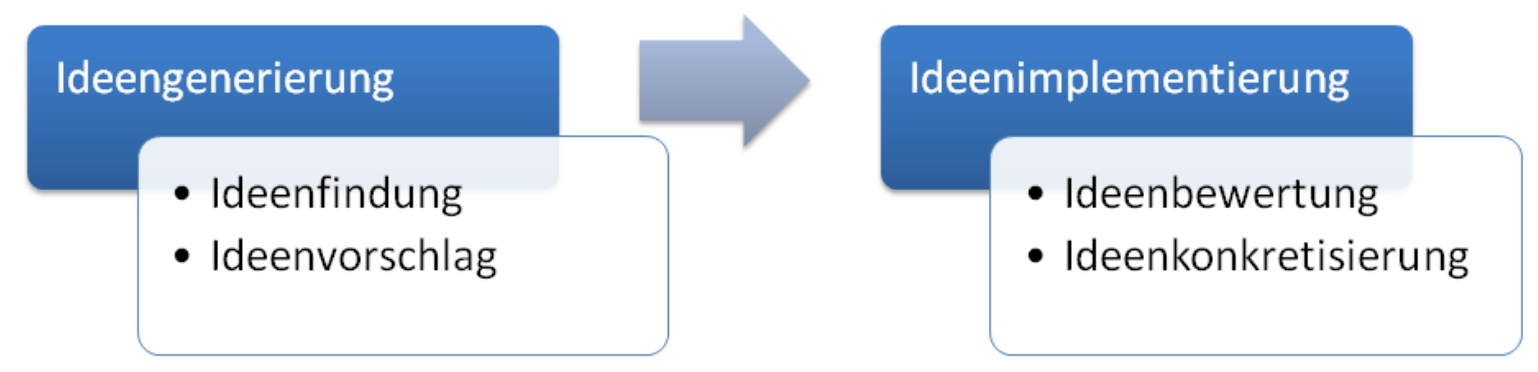

Abbildung 3: Der Ideenevolutionsprozess innerhalb der GENIE-Community (Quelle: Eigene Darstellung)

\subsection{Forschungsmethodik und Realisierung}

Im Rahmen des Forschungsprojektes GENIE sollen derartige Innovationscommunities pilotiert und dafür geeignete Methoden, Modelle und Werkzeuge entwickelt werden, die die Community von der Ideenfindung bis zur Ideenimplementierung sowohl im Internet als auch in der realen Welt durchgängig unterstützen. Abbildung 4 veranschaulicht, dass bei diesem Forschungsansatz die zu initiierende Community stufenweise geplant und entwickelt wird, wodurch es zu einer steten Realisierung von Verbesserungspotenzialen kommt. Die Methodik der Pilotierung ermöglicht dabei die Entwicklung und Implementierung sozio-technischer Innovationen in ihrem natürlichen Umfeld. Durch diesen methodischen Innovationsprüfstein wird eine hohe externe Validität der Ergebnisse gewährleistet sowie ein effizienter und effektiver Erkenntnistransfer in die Praxis ermöglicht (Schwabe, Krcmar 2000; Witte 1997).

Im Kontext der Pilotierung kommt es im Rahmen des Community Engineerings (Leimeister, Krcmar 2006) zur Entwicklung und Implementierung einer geeigneten Internetplattform, die es den Community-Mitgliedern ermöglicht, über das Internet kollaborativ zu agieren. Dies befördert die Entwicklung sozialer Beziehungen der Community-Mitglieder untereinander. 


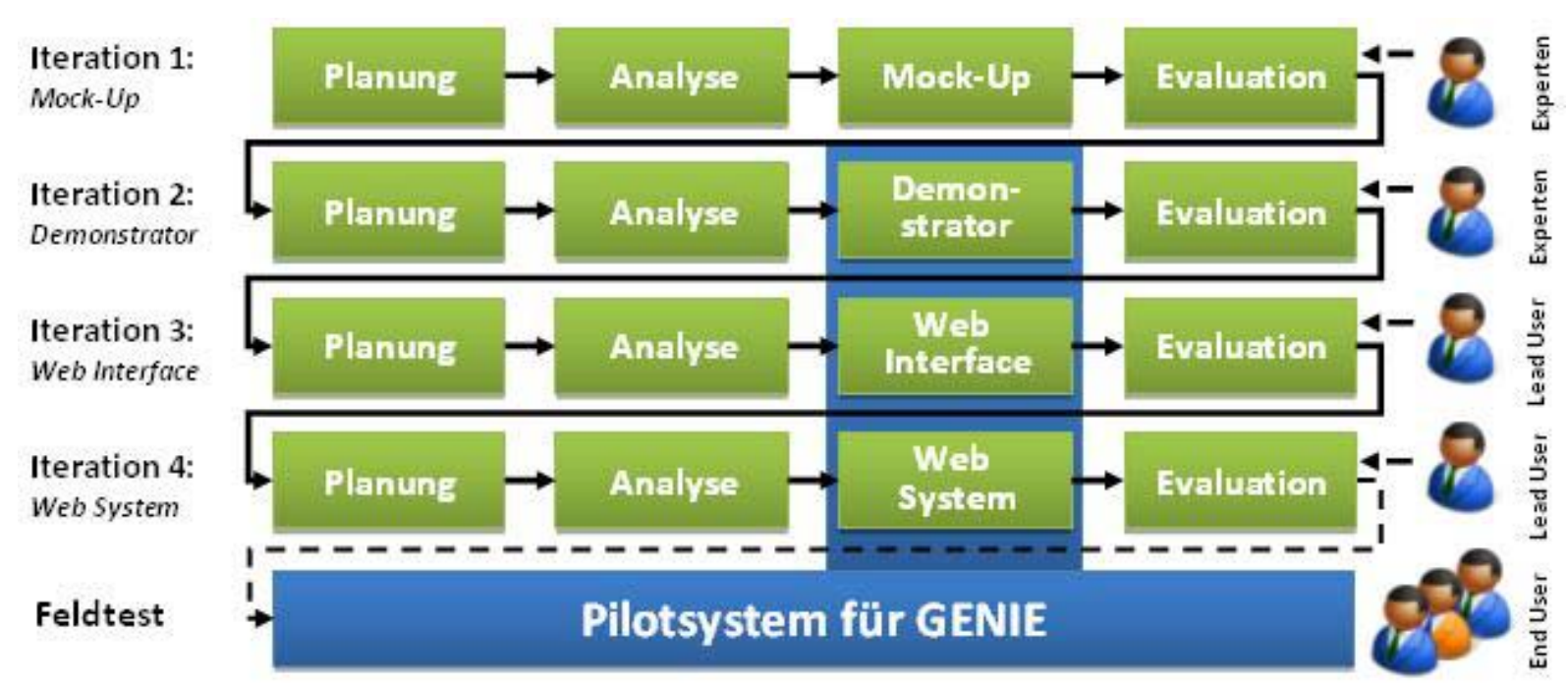

Abbildung 4: Der iterative Entwicklungsprozess der GENIE-Community Plattform(Quelle: Eigene Darstellung)

Als Community-unterstützendes Element fungieren hierbei IT-gestützte Ideenwettbewerbe. Ideenwettbewerbe erlauben es dem Unternehmen eine bestehende Community zu aktivieren, da für potenzielle Community-Mitglieder zusätzliche Anreize geschaffen werden, sich aktiv an der Generierung neuer Produktideen zu beteiligen (Ebner et al. 2010). Vielversprechend sind darüber hinaus innovative Benutzerschnittstellen der Community-Plattform. Durch die Entwicklung und Implementierung des IdeaMirror ${ }^{\mathrm{TM}}$ - ein Werkzeug, das die Darstellung von Ideen auf großen Wandbildschirmen ermöglicht - kann die gemeinschaftsgestützte Innovationsentwicklung aber auch aus den Internet in die „physische“ Welt getragen werden. So können in geeigneten öffentlichen Räumen, wie z.B. auf Messen, Foyers, etc., die Online in der Community entstandenen Ideen eingesehen und bewertet werden. Dadurch werden die Ideen einer größeren Zielgruppe präsentiert, deren Diskussion und Verfeinerung gefördert sowie die Vernetzungsaktivitäten der Ideengeber vereinfacht (Koch, Möslein 2007).

\subsection{Nutzen des GENIE-Ansatzes}

Durch das Forschungsprojekt GENIE ergibt sich für Softwareunternehmen eine Vielzahl direkter Nutzenpotenziale. Es kommt zu einer Systematisierung des Innovationsmanagements von Softwareunternehmen, so dass ein vertieftes Verständnis für die Innovationsentwicklung in der Softwareindustrie entwickelt werden kann und die Communities direkt an die spezifischen Anforderungen der Softwareentwicklung angepasst werden können. Durch die GENIE-Communities können vorher ungenutzte Innovationspotenziale von Kunden und anderen Wertschöpfungspartnern gehoben werden, was zu einer Verbesserung der Innovationsfähigkeit der initiierenden Unternehmen führt.

Im Rahmen des Forschungsprojektes kommt es zu einer induktiven Generierung und Erprobung neuartiger Ansätze, Methoden und Werkzeuge im Sinne des OpenInnovation-Gedanken über die Pilotierung der sozio-technischen Innovation der "GENIE Innovation Community“. Die Endergebnisse werden zu einem unternehmensgrenzen überschreitendem und gemeinschaftsgestütztem Innovationsmanage- 
mentmodell zusammengeführt und können so ein wesentlichen Beitrag zur Open Innovation Forschung liefern.

\section{$4 \quad$ Zukünftiger Forschungsbedarf}

Da im Bereich der Innovationscommunities lediglich auf erste wissenschaftliche Erkenntnisse, wie z.B. aus dem Community Engineering und der Open Innovation Forschung, zurückgegriffen werden kann, ergibt sich auf Basis der GENIE-Ergebnisse weiterer Forschungsbedarf in vielfältigen Ausprägungen.

Aus theoretischer Sicht besteht Bedarf nach fundierten und empirisch stützbaren Theorien, Modellen, Methoden und Werkzeugen für ein gemeinschaftsgestütztes Innovationsmanagement. Bereits vorhandene, punktuelle Erkenntnisse aus unterschiedlichen Forschungsbereichen müssen hierfür konsolidiert und schrittweise erweitert werden. Es muss erarbeitet werden, welche Einflussfaktoren und Rahmenbedingungen den Erfolg bzw. Misserfolg von unternehmensübergreifenden, gemeinschafsgestützten Innovationsvorhaben beeinflussen. Grundsätzliche Ursache-Wirkungszusammenhänge und deren Stärken und Richtungen sind weder konzeptionell noch empirisch umfänglich erforscht. Es sind Erkenntnisse darüber zu gewinnen, für welche Art von Produkten und in welchen Industrien Open Innovation als Wertschöpfungsstrategie eine sinnvolle Ergänzung zum klassischen Innovationsmanagement darstellen kann - und wo nicht.

Aus sozio-ökonomischer Sicht sind grundlegende Fragen der Aufbau- und Ablauforganisation bei gemeinschaftsgestützter Innovationsentwicklung zu beantworten. Teile von Innovationsaufgaben müssen in Zukunft so gestaltet werden, so dass sie einerseits gemeinschaftlich von einer großen Zahl von beteiligten Wertschöpfungspartnern unabhängig und wohlmöglich gleichzeitig bearbeitet und die Artefakte dieser Tätigkeiten andererseits - mittels des Einsatzes von Informationstechnologie - in intelligenter Weise zu einem innovativem, marktfähigen Endprodukt zusammengesetzt werden können. Es ist allerdings noch nicht geklärt, welche Teilaufgaben unter welchen Rahmenbedingungen, wann besser im Open Innovation Ansatz und wann im Closed Innovation Ansatz bearbeitet werden sollen. Hieran anknüpfend ergeben sich außerdem weitreichende neue Forschungsfelder in Bezug auf Führung, Motivation und Kompetenzentwicklung der beteiligten Mitarbeiter. Um das ganze Potential der interaktiven Wertschöpfung ausnutzen zu können, muss zukünftige Forschung den Blick aber auch verstärkt auf die unternehmensexternen Community-Mitglieder richten, da das Potential von offenen Innovationsprozessen vor allem in der Kollaboration der beteiligten Wertschöpfungspartner liegt (Franke, Shah 2003; GascóHernández, Torres-Coronas 2004; Nemiro 2001; Sawhney et al. 2005; Wagner, Prasarnphanich 2007). Das Schaffen eines eingehenden Verständnisses für die Prinzipien der kollaborativen Innovationsentwicklung ist daher von zentraler Bedeutung, um das ganze Potential offener Innovationsprozesse nutzen zu können. Es muss ein vertieftes Verständnis für die Erwartungen, Motive und Handlungen der beteiligten Wertschöpfungsgruppen geschaffen werden, um entsprechende Anreizmechanismen entwickeln und bspw. "Social Capital“ aufbauen zu können (Leimeister et al. 2009). Hier besteht sowohl Bedarf nach einer grundlegenden Analyse der sozialen Interaktionen als auch der (sozialen) Netzwerkbildung innerhalb der Innovationscommunities. Vor allem in der Softwareentwicklung steht die Forschung an dieser Stelle aber noch am Anfang ihrer Entwicklung (Holl et al. 2006). 
Aus technischer Perspektive sind ubiquitäre Zugänge durch ubiquitäre Benutzerschnittstellen oder mobile Endgeräte ebenso denkbar wie neue (mobile bzw. kontextsensitive) Dienste. In diesem Zusammenhang ist insbesondere die Untersuchung und Entwicklung von Technologien bedeutungsvoll, die die Community-Mitglieder in ihren Vernetzungs- und Kollaborationsaktivitäten unterstützen sowie der gemeinschaftsgestützten Ablauf- und Arbeitsorganisation dienen können. Die Entwicklung von konkreten Empfehlungen zur Gestaltung und Implementierung von Instrumenten zur Unterstützung entsprechender Aktivitäten entlang des Innovationsprozesses ist in diesem Kontext ein weiterer erfolgsversprechender Ansatz. Außerdem fehlen Arbeiten zu sinnvollen Unterstützungstools für das effiziente und effektive CommunityManagement.

Aus juristischer Sicht tangiert interaktive Wertschöpfung zwangsläufig aber auch immer die Fragestellung nach den bei der gemeinschaftlichen Innovationsentwicklung entstehenden Urheber- und Eigentumsrechten. Obwohl Open Innovation in der unternehmerischen Praxis eine immer wichtigere Rolle spielt, konnte diesbezüglich bis zum heutigen Zeitpunkt noch keine befriedigende Lösung gefunden werden.

In methodischer Hinsicht ist eine Weiterentwicklung der Methodik der Pilotierung notwendig, um für ähnliche Forschungssituationen über ein stärker erprobtes, valides und reliables Forschungsvorgehen zu verfügen. Das vorgestellte Forschungsprojekt GENIE kombiniert bestehende Ansätze der Wirtschaftsinformatik zur Pilotierung von sozio-technischen Innovationen und wendet diese auf die spezifische Situation der gemeinschaftsgestützten Innovationentwickling an, dies kann aber nur ein erster Schritt auf dem Weg der Methodenentwicklung sein.

Forschung zu gemeinschaftsgestützten Innovationsentwicklung muss eine Integration bereits existierender Erkenntnisse und Ansätze aus unterschiedlichen Wissenschaftsdisziplinen leisten. Diese Integrationsperspektive anzubieten und diese Weiterentwicklungsleistung zu erbringen ist Chance und Herausforderung für die Wirtschaftsinformatik ebenso wie für die Arbeits- und Dienstleistungsforschung.

\section{Literatur}

Boston Consulting Group (2006): Innovationsstandort Deutschland - Quo vadis?

Bretschneider, U.; Huber, J. M.; Leimeister, J. M.; Krcmar, H. (2008): Community for Innovations: Developing an Integrated Concept for Open Innovation. Vorgestellt auf der International Federation for Information Processing (IFIP8.6), Madrid, S. 503-510.

Bretschneider, U.; Leimeister, J. M.; Krcmar, H. (2009): Methoden der Kundenintegration in den Innovationsprozess: Eine Bestandsaufnahme. Lehrstuhl für Wirtschaftsinformatik, Technische Universität München.

Chesbrough, H. W. (2003): The era of open innovation. In: MIT Sloan Management Review, Vol. 44 (2003) Nr. 3, S. 35-41.

Chesbrough, H. W. (2007): Why companies should have open innovation business models. In: MIT Sloan Management Review, Vol. 48 (2007) Nr. 2, S. 22-28. 
Chesbrough, H. W.; Schwartz, K. (2007): Innovationg business models with codevelopment partnerships. In: Research-Technology Management, Vol. 50 (2007) Nr. 1, S. 55-59.

Ebner, W.; Leimeister, J. M.; Krcmar, H. (2010): Community Engineering for Innovations: The Ideas Competition as a method to nurture a Virtual Community for Innovations. In: R \& D Management, Vol. 40 (2010) Nr. in press.

Franke, N.; Shah, S. (2003): How communities support innovative activities: an exploration of assistance and sharing among end-users. In: Research Policy, Vol. 32 (2003) Nr. 1, S. 157-178.

Gascó-Hernández, M.; Torres-Coronas, T. (2004): Virtual teams and their search for creativity. In: Godar, S. H.; Pixy Ferris, S. (ed.): Virtual and collaborative teams Hershey: PA: Idea Group.

Gassmann, O.; Enkel, E. (2004): Towards a theory of open innovation: Three core process archetypes. Vorgestellt auf der R\&D Management Conference (RADMA), Lissabon.

Holl, F. L.; Menzel, K.; Morcinek, P.; Mühlberg, J. T.; Schäfer, I.; Schüngel, H. (2006): Studie zum Innovationsverhalten deutscher SoftwareEntwicklungsunternehmen, Berlin: Eigenverlag.

Koch, M.; Möslein, K. (2007): Diskontinuierliche Innovation fördern: Die Rolle von Idea Mirrors zur Unterstützung von Innovation und Kooperation im Unternehmen. Vorgestellt auf der Wirtschaftsinformatik 2007, Karlsruhe, S. 787-804.

Lakhani, K. R.; von Hippel, E. (2003): How open source software works: "free" userto-user assistance. In: Research Policy, Vol. 32 (2003) Nr. 7, S. 923-943.

Leimeister, J. M.; Huber, M.; Bretschneider, U.; Krcmar, H. (2009): Leveraging Crowdsourcing - Theory-driven Design, Implementation and Evaluation of Activation-Supporting Components for IT-based Idea Competitions. In: Journal of Management Information Systems, Vol. 26 (2009) Nr. 1, in press.

Leimeister, J. M.; Krcmar, H. (2006): Community-Engineering: Systematischer Aufbau und Betrieb Virtueller Communitys im Gesundheitswesen. In: Wirtschaftsinformatik, Vol. 48 (2006) Nr. 6, S. 418-429.

Lévy, P. (1997): Collective Intelligence: Mankinds emerging world in cyberspace, New York

Nemiro, J. E. (2001): Connection in creative virtual teams. In: Journal of Behavioral and Applied Management, Vol. 2 (2001) Nr. 2, S. 92-112.

Piller, F. T. (2003): Von Open Source zu Open Innovation. In: Harvard Business Manager, Vol. 25 (2003) Nr. 12, S. 114.

Reichwald, R.; Piller, F. (2006): Interaktive Wertschöpfung, Wiesbaden: Gabler. 
Sawhney, M.; Verona, G.; Prandelli, E. (2005): Collaborating to Create: The Internet as a Platform for Customer Engagement in Product Innovation. In: Journal of Interactive Marketing, Vol. 19 (2005) Nr. 4, S. 4.

Schwabe, G.; Krcmar, H. (2000): Piloting a Social-technical Innovation. Vorgestellt auf der 8th European Conference on Information Systems (ECIS), Vienna, S. 132-139.

Surowiecki, J. (2005): The wisdom of crowds, New York: Anchor Books.

von Hippel, E. (1978): Succesful industrial products from customer ideas: presentation of a new customer-active paradigm with evidence and implication. In: Journal for Marketing, Vol. 42 (1978) Nr. 1, S. 39-49.

von Hippel, E. (1994): "Sticky Information" and the locus of problem solving: Implications for innovation. In: Management Science, Vol. 40 (1994) Nr. 4, S. 429-439.

von Hippel, E. (2005): Democratizing innovation, Cambridge, Mass.: MIT Press.

von Krogh, G.; Spaeth, G.; Lakhani, K. R. (2003): Community, joining, and specialization in open source software innovation: a case study. In: Research Policy, Vol. 32 (2003) Nr. 7, S. 121.

Wagner, C.; Prasarnphanich, G. (2007): Innovating Collaborative Content Creation: The Role of Altruism and Wiki Technology. Vorgestellt auf der 40th Hawaii International Conference on System Sciences (HICCS), Big Island, Hawaii.

Witte, E. (1997): Feldexperimente als Innovationstest - Die Pilotprojekte zu neuen Medien. In: Zeitschrift für betriebswirtschaftliche Forschung, Vol. 49 (1997) Nr. 5, S. 419-436.

Wohlrab, O. (2003): IT-Innovationen als Schlüssel für Wirtschaftswachstum. Initi@tive D21: "Neue Jobs durchs Internet. Impulse für Wachstum und Wettbewerbsfähigkeit in der Informationsgesellschaft", S. 82.

\section{Autor:}

Leimeister, Jan Marco, Prof. Dr. Universität Kassel Lehrstuhl für Wirtschaftsinformatik Nora-Platiel-Str. 4, 34127 Kassel leimeister@uni-kassel.de

www.wi-kassel.de 